\section{Tamanho de amostra e estimativa de custo em levantamento epidemiológico de cárie dentária}

\section{Sample size and costs estimate in epidemiological survey of dental caries}

Maria Lucia Bellon'

Gláucia Maria Bovi Ambrosano'

Stela Márcia Pereira"'I

Sílvia Helena de Carvalho Sales-Peres"

Marcelo de Castro Meneghim'

Antonio Carlos Pereira'

Elaine Pereira da Silva Tagliaferro, ${ }^{1, v}$

Vanessa Pardi'

' Departamento de Odontologia Social da Faculdade de Odontologia de Piracicaba da Universidade Estadual de Campinas, SP.

" Área de Epidemiologia e Saúde Pública DMV - Setor de Preventiva da Universidade Federal de Lavras, Lavras, MG.

III Departamento de Odontopediatria, Ortodontia e Saúde Coletiva da Faculdade de Odontologia de Bauru, da Universidade de São Paulo, Bauru, SP.

IV Departamento de Odontologia Social da Faculdade de Odontologia de Araraquara - UNESP, SP.

\section{Resumo}

O objetivo do presente estudo foi analisar como a prevalência e a distribuição da cárie dentária influenciam o tamanho da amostra em levantamentos epidemiológicos, e os custos para sua realização. Foram utilizados dados de levantamentos realizados em escolares de 12 anos em Bauru nos anos de 1976, 1984, 1990, 1994 e 2001, e em Piracicaba nos anos de 2001 e 2005. Os tamanhos amostrais foram dimensionados considerando-se a média e o desvio padrão obtidos, fixando-se erro amostral em $1 \%$, $2 \%, 5 \%$ e $10 \%$. Os custos foram estimados considerando material permanente, de consumo e recursos humanos. Verificouse aumento no tamanho das amostras em ambos os municípios, variando de 119 em 1976 para 1.118 em 2001 em Bauru, e de 954 em 2001 para 1.252 em 2005 em Piracicaba, considerando-se um erro amostral de $10 \%$. Considerando-se diferentes erros amostrais, verificou-se o custo para o levantamento, sendo que o mesmo depende do quanto o pesquisador se permite errar em relação ao verdadeiro valor da média da população. Conclui-se que a diminuição da prevalência da cárie dentária determinou o aumento no tamanho das amostras e a elevação dos custos para realização dos levantamentos.

Palavras-chave: Cárie dentária. Epidemiologia. Tamanho de amostra. Prevalência. Estudos Transversais. Custos e Análise de custo. 


\section{Abstract}

This study aimed to analyze how the prevalence and the distribution of dental caries influence the sample size in epidemiological surveys, and how much are the costs. Secondary data of oral health surveys in 12-year-old schoolchildren from Bauru in 1976, 1984, 1990, 1994, and 2001, and from Piracicaba in 2001 and 2005 were studied. Sample sizes were estimated taking into account the mean DMFT and standard deviation of each survey, establishing sampling errors of $1 \%, 2 \%, 5 \%$, and $10 \%$. Costs were estimated considering permanent material, consumption material and human resources. The sample size in both towns needed to be increased, ranging from 119 in 1976 to 1,118 in 2001 in Bauru, and from 954 in 2001 to 1,252 in 2005 in Piracicaba, when a sampling error of $10 \%$ was considered. The cost of dental caries surveys was verified considering different sampling errors. This cost depends on how acceptable is the margin of difference between the true mean and the one found in the survey. In conclusion, the reduction in the prevalence of dental caries has determined the need for increase in sample size and in costs for conducting the surveys.

Keywords: Dental caries. Epidemiology. Sample size. Prevalence. Cross-sectional studies. Costs and cost analysis.

\section{Introdução}

A cárie dentária ainda é considerada como um dos principais problemas de saúde bucal, em termos de Saúde Pública no Brasil ${ }^{1}$, sendo também considerada como a maior causa de perda dentária em todo o mundo ${ }^{2}$. Entretanto, a partir da década de 70 tem sido constatado um declínio na prevalência da doença, inicialmente observado em países desenvolvidos ${ }^{3,4} \mathrm{e}$, posteriormente, em países em desenvolvimento como o Brasil ${ }^{5,6}$.

Quatro grandes levantamentos de base nacional realizados em 1986, 1996, 2003 e 2010 apontaram um expressivo declínio na prevalência de cárie em crianças de 12 $\operatorname{anos}^{7-10}$, principalmente nas regiões Sul e Sudeste. ${ }^{9}$ No entanto, essa redução não tem ocorrido de modo homogêneo, havendo concentração da doença em uma pequena parcela da população ${ }^{11,12}$.

De acordo com as recomendações da Organização Mundial de Saúde (OMS), autoridades sanitárias devem promover a realização periódica de levantamentos epidemiológicos das doenças bucais para o conhecimento das condições de saúde da população, a fim de planejar, executar e avaliar ações de controle dos níveis da doença ${ }^{13}$. A maioria dos levantamentos epidemiológicos é realizada em uma amostra, que deve ser representativa da população a ser estudada. Em contrapartida, um dos mais frequentes questionamentos se refere ao tamanho dessa amostra com o objetivo de se obter um estudo confiável ou significativo. Para o cálculo do tamanho da amostra devem ser levados em consideração alguns itens, tais como tipo de estudo, erro tolerado, nível de significância complementar à confiança desejada, poder e tamanho da população. Para os levantamentos epidemiológicos, o cálculo do tamanho da amostra depende de dados de estudos similares prévios e, na falta destes, torna-se necessária a realização de uma "fase piloto" ${ }^{14}$.

A literatura apresenta vários estudos sobre cálculo do tamanho da amostra. A maneira de se obter esse cálculo depende do tipo de pesquisa realizada, devendo-se 
considerar o tipo de variável estudada. Para a variável quantitativa trabalha-se com média e desvio padrão, enquanto a variável qualitativa requer proporção e desvio padrão ${ }^{15}$. Para estudos experimentais utiliza-se o erro tipo I e o erro tipo $\mathrm{II}^{16}$. Estudos que utilizam amostra do tipo probabilística citam nível de significância, intervalo de confiança e prevalência como itens para cálculo do tamanho da amostra ${ }^{17,18}$.

Segundo Roncalli et al. ${ }^{19}$ (1998), a OMS recomenda que o tamanho da amostra em cada grupo etário varie de 25 a 50, dependendo da prevalência e severidade da doença, levando-se em consideração o nível de confiança, as frequências de ausência e presença da doença, a variabilidade e a margem de erro aceitável.

A definição do tamanho da amostra em estudos epidemiológicos é difícil tanto pelos aspectos técnicos como pela relação fidedignidade versus factibilidade, pois se sabe que amostras maiores, embora gerem maior confiabilidade, aumentam os custos da pesquisa ${ }^{20}$.

Apesar de vários autores serem unânimes quanto ao fato de que amostras pequenas podem comprometer o resultado e amostras grandes podem encarecer o custo da pesquisa, pouca informação se conhece sobre gastos reais na realização de levantamentos epidemiológicos. Na área médica essa preocupação já pode ser observada em estudos que estimaram custos para o sistema de saúde no setor público com procedimento clínicos, hospitalares e cirúrgicos ${ }^{21,22}$.

Dessa forma, o presente estudo tem como objetivo analisar como a mudança da prevalência da cárie dentária e a variabilidade entre as crianças influenciam o tamanho necessário da amostra e, consequentemente, o custo do levantamento por parte dos Serviços Públicos.

\section{Material e Métodos}

\section{Coleta de dados}

Foram utilizados dados de estudos prévios realizados nas cidades de Piracicaba em 2001 e 2005, e Bauru nos anos de 1976, 1984, 1990, 1995 e 2001. Para os levantamentos do município de Piracicaba os tamanhos amostrais foram calculados com base na experiência de cárie em estudos prévios. As escolas foram selecionadas por meio de amostragem probabilística por conglomerados a partir da lista das escolas fornecida pela Diretoria de Ensino - Região de Piracicaba, contemplando-se de maneira proporcional os alunos de 12 anos de idade matriculados nas escolas da rede pública $\mathrm{e}$ privada.

Os estudos mais antigos, provenientes do município de Bauru, foram considerados passíveis de inclusão na presente pesquisa devido à amostra selecionada ter alcançado a proporção sugerida pela Organização Mundial de Saúde em 1991 ${ }^{13}$, que recomenda, para cada grupo etário, de 25 a 50 indivíduos, dependendo da prevalência e severidade da doença, sendo este o limite mínimo admitido para populações com baixos índices de cárie. Embora esta metodologia tenha sido publicada posteriormente aos estudos de 1976, 1984 e 1990, o tamanho amostral mínimo foi alcançado. Em todos os levantamentos, os elementos amostrais foram divididos proporcionalmente entre os gêneros masculino e feminino, bem como as escolas foram escolhidas aleatoriamente por região da cidade, com o intuito de delinear o quadro de saúde bucal dos escolares de 12 anos de idade da cidade de Bauru-SP da maneira mais fiel possível, guardadas as limitações de estudos com base em amostras populacionais. Em 1976, 1984, 1990, 1995 e 2001, foram examinadas 261 crianças provenientes de seis escolas, 321 crianças de oito escolas, 253 crianças de 10 escolas, 377 crianças de 23 escolas e 211 crianças de nove escolas, respectivamente ${ }^{6}$.

Os exames foram realizados seguindo as recomendações da $\mathrm{OMS}^{13}$. Entretanto, para os estudos realizados de 1976 a 1995 no município de Bauru, foi utilizada a sonda exploradora $\mathrm{n}^{\circ} 23$. Nos estudos mais recentes, a sonda "Ball Point" (sonda IPC) foi utilizada ${ }^{23}$. Os examinadores foram previamente calibrados em todos os estudos, 
sendo que em Bauru foi utilizada a técnica do consenso (maior que $90 \%$, para os levantamentos de 1995 e 2001) e em Piracicaba foi atingido Kappa $>0,91$ para intra e inter-examinador ${ }^{6,12}$. Para os levantamentos anteriores, em Bauru, não há relatos sobre o grau de concordância.

\section{Cálculo do Tamanho da Amostra}

Em levantamentos de saúde bucal, geralmente o pesquisador tem como objetivo estimar a média $(\mu)$ do índice CPO-D (número de dentes cariados, perdidos e obturados) de uma determinada população. Essa média é estimada utilizando-se, na maioria das vezes, informações provenientes de uma amostra, pois as populações a serem estudadas são grandes, o que tornaria o estudo inviável devido aos custos e ao tempo.

$\mathrm{O}$ número que representa o valor mais provável da média (baseado nos dados amostrais) é chamado de estimativa pontual de $\mu$. Como o valor estimado na maior parte das vezes não é exatamente igual à média verdadeira, determina-se um intervalo de confiança que representa um intervalo de valores prováveis para a média baseado nos dados amostrais.

Assim, um intervalo de confiança de 95\% para a média do CPO-D representa um intervalo no qual se tem $95 \%$ de confiança da cobertura do verdadeiro valor da média do CPO-D da população.

O nível de confiança deve ser fixado de acordo com a probabilidade de acerto que se deseja obter na estimação da média do CPO-D.

Assim, fixando-se o valor da semi-amplitude do Intervalo de confiança e o nível de confiança, pode-se calcular o tamanho da amostra pela expressão:

$$
n=\left(\frac{t_{(n-1 ; 0,05)^{S}}}{d}\right)^{2}
$$

onde t é obtido da tabela de distribuição t, d é a semi-amplitude do Intervalo de confiança, s é uma estimativa do desvio padrão do CPO-D na população e n o tamanho da amostra.
Assim, utilizando-se essa expressão, foram dimensionadas amostras para os levantamentos realizados em Piracicaba e Bauru, levando-se em consideração a média e o desvio padrão obtidos nestes estudos, fixando-se o erro amostral (d) em $1 \%, 2 \%$, $5 \%$ e $10 \%$ da média.

Como nos levantamentos foram utilizados sorteios por estágio, foi realizada a correção para o "efeito de delineamento", multiplicando-se o tamanho amostral encontrado por dois, de acordo com o Projeto SB-Brasil 20039.

O tamanho da amostra foi ainda corrigido considerando-se o número de crianças na população.

A população de 12 anos nos municípios de Bauru e Piracicaba está apresentada na Tabela 1, de acordo com censo demográfico realizado pelo IBGE nos anos de 1970, 1980, 1991,1996 e $2000^{24}$.

Após o dimensionamento das amostras em função da variação do CPO-D no decorrer do tempo e dos erros amostrais, os custos para os levantamentos de cárie atuais foram estimados considerando-se os preços dos materiais permanentes, de consumo e recursos humanos.

\section{Cálculo dos Custos}

Para o cálculo dos custos foi suposto que em cada período foram examinados cerca de 60 escolares. Os profissionais considerados foram um cirurgião-dentista (CD) para proceder ao exame bucal e duas auxiliares de consultório dentário (ACDs), sendo uma com função de anotador dos resultados e outra para a organização das crianças e dos materiais. Os materiais permanentes (sonda ball point/IPC, espelho com cabo e caixa de inox) foram considerados como custo fixo, pois esses materiais podem ser utilizados em diversos levantamentos. Não foram computados valores para estufa ou autoclave, considerando-se que esse tipo de equipamento faz parte das unidades dos serviços públicos. Também não foi feita referência ao transporte (carro e combustível) para o deslocamento dos profissionais 
Tabela 1 - Total de crianças com 12 anos de idade nos municípios de Bauru e Piracicaba de acordo com IBGE.

Table 1 - Number of 12-year-old schoolchildren in Bauru and Piracicaba according to IBGE.

\begin{tabular}{lcc}
\hline Cidade & Ano & População de 12 anos \\
\hline BAURU & 1970 & 3.371 \\
& 1980 & 3.750 \\
& 1991 & 5.279 \\
& 1996 & 5.598 \\
PIRACICABA & 2000 & 5.461 \\
& 1996 & 6.012 \\
\hline
\end{tabular}

Fonte/Source: IBGE [Acessado em 19 de janeiro de 2008]

às escolas devido ao fato de as Prefeituras disponibilizarem condução própria para tais trabalhos.

O custo do profissional CirurgiãoDentista foi calculado, mediante a Lei $\mathrm{n}^{\circ}$ 3.999 de 15 de dezembro de 1961, incluindo valores de salário, insalubridade, décimo terceiro, férias e encargos sociais, chegando ao valor da hora profissional. Para a ACD, também foram feitos os mesmos cálculos, porém o piso salarial foi sugerido pelo Sindicato dos Odontologistas, pois não existe representação sindical específica para essa categoria (Tabela 2). Na Tabela 3 é possível verificar os custos detalhados (material de consumo, material permanente e recursos humanos) para avaliação de 60 crianças no período de 4 horas.

\section{Resultados}

Verifica-se, pela Tabela 4, que no município de Bauru, a média e desvio padrão (dp) variaram de 9,9 $(4,0)$ em 1976 para 1,5 $(2,1)$ em 2001, sendo que o tamanho da amostra deveria ser de 430 e 2.771, respectivamente, considerando erro amostral de 5\%. Para erro amostral de $10 \%$, o tamanho da amostra seria de 119 e 1.118 nos respectivos anos. Em Piracicaba, a média e o desvio padrão (dp) variaram de 1,7 $(2,1)$ em 2001 para 1,3 $(1,9)$ em 2005. O tamanho da amostra deveria ser

Tabela 2 - Demonstrativo sobre cálculo da hora profissional do Cirurgião-Dentista e da ACD.

Table 2 - Hourly rate calculation for a Dental Surgeon and for a Dental Assistant.

\begin{tabular}{|c|c|c|}
\hline Valores referentes ao período de um mês* & Cirurgião-dentista & $A C D$ \\
\hline Salário & $\begin{array}{c}\mathrm{R} \$ 1.635,00 \\
\text { (três salários mínimos) }\end{array}$ & $\mathrm{R} \$ 618,08$ \\
\hline Insalubridade ( $40 \%$ do salário) & $\mathrm{R} \$ 654,00$ & $\mathrm{R} \$ 247,23$ \\
\hline Cesta Básica & $\mathrm{R} \$ 0,0$ & $\mathrm{R} \$ 270,44$ \\
\hline Décimo terceiro (1/12) & $\mathrm{R} \$ 136,25$ & $\mathrm{R} \$ 51,51$ \\
\hline Férias & $\mathrm{R} \$ 253,81$ & $\mathrm{R} \$ 78,01$ \\
\hline $\begin{array}{l}\text { Encargos trabalhistas (INSS e FGTS) } 35,80 \% \text { do salário } \\
\text { INSS }=27,8 \% \text { do salário } \\
\text { FGTS }=8 \% \text { do salário (salário + insalubridade) }\end{array}$ & $\mathrm{R} \$ 1170,66$ & $\mathrm{R} \$ 442,55$ \\
\hline Total & $\begin{array}{c}\text { R\$ 3.849,67 } \\
\text { (100 horas/mês) }\end{array}$ & $\begin{array}{c}\text { R\$ } 1.707,82 \\
\text { (200 horas/mês) }\end{array}$ \\
\hline Valor/hora & $\mathbf{R} \$ \mathbf{3 8 , 5 0}$ & $\mathrm{R} \$ \mathbf{8 , 5 4}$ \\
\hline
\end{tabular}

* Valores atualizados conforme salário mínimo vigente em 2011 / Figures updated according to minimum wage in effect in 2011 
Tabela 3 - Itens considerados para o cálculo dos custos de um levantamento epidemiológico em Cárie Dentária. Table 3 - Items taken into account to calculate the costs of a dental caries epidemiological survey.

\begin{tabular}{lll}
\hline Itens & Descrição & Custos \\
\hline Material permanente (custo fixo) & sonda ball point/IPC, espelho com cabo e caixa de inox & $\mathrm{R} \$ 1.456,58$ \\
\hline Material de consumo* & Máscara, luvas, espátula de madeira, recipiente plástico para & $\mathrm{R} \$ 74,48$ \\
& descarte dos instrumentais, álcool, gorros, bucha, detergente, \\
& algodão, gaze, papel toalha, toalha de tecido, saco de lixo, \\
& lápis, borracha, apontador, caneta, clipes, grampeador, \\
& planilhas para preenchimento de dados, envelope, \\
& embalagem de autoclave com adesivo, glutaraldeído & \\
\hline Recursos humanos* & 1 Cirurgião-dentista & $\mathrm{R} \$ 154,00$ \\
& 2 Auxiliares de consultório dentário & $\mathrm{R} \$ 68,32$ \\
\hline
\end{tabular}

*Considerando-se o exame de 60 crianças examinadas em um período de $4 \mathrm{~h} /{ }^{*}$ Considering examination of 60 children seen in a period of $4 \mathrm{~h}$.

de 2.564 e 3.052 em 2001 e 2005, respectivamente, considerando erro amostral de $5 \%$, sendo que para erro amostral de $10 \%$ o tamanho da amostra seria de 954 e 1.252, respectivamente.

De acordo com a Tabela 5, em relação aos custos para realização de um levantamento epidemiológico atual de cárie dentária, considerando-se erro amostral de 5\%, no município de Bauru foi estimado o custo de R\$15.163,80 e no município de Piracicaba o custo estimado foi de $\mathrm{R} \$ 16.553,81$. Para erro amostral de 10\%, aceitável neste tipo de estudo, o custo calculado para o município de Bauru seria de $\mathrm{R} \$$ 6.986,95 e em Piracicaba seria de $\mathrm{R} \$ 7.649,81$. Os valores mencionados se referem ao custo total (materiais de consumo, recursos humanos e materiais permanentes).

\section{Discussão}

Estudos epidemiológicos têm demonstrado expressivo declínio da prevalência da cárie dentária em escolares de 12 anos em diferentes países do mundo ${ }^{3,5}$. O levantamento de base nacional, realizado em 2003, SB-BRASIL ${ }^{9}$, mostrou redução do índice CPO-D principalmente nas regiões Sul e Sudeste do país. Contudo, a redução da cárie dentária tem apresentado tendências que indicam o fenômeno da polarização, revelando desigualdades na distribuição da doença, com a concentração da mesma em pequenos grupos dentro de uma mesma população ${ }^{12,25}$. Dessa forma, torna-se importante aos gestores dos Serviços Públicos conhecerem essas informações a fim de se construir projetos e programas que atuem efetivamente na área da saúde ${ }^{25}$.

Assim, os levantamentos epidemiológicos constituem fonte de dados importantes sobre as condições de saúde de uma população e devem ser realizados periodicamente pelas Instituições Públicas, de acordo com recomendações da $\mathrm{OMS}^{13}$. No entanto, a realização desses levantamentos nem sempre é uma tarefa fácil de ser realizada, pois metodologia específica e padronização são requisitos necessários para a confiabilidade dos resultados encontrados $^{19}$. Adicionalmente, o tamanho da amostra é fundamental para a validade dos resultados e sempre constitui um dos grandes questionamentos pelos idealizadores da pesquisa.

Vários autores ressaltaram a importância do cálculo do tamanho da amostra e descreveram os elementos influentes na sua determinação, como a necessidade de se definir as variáveis quantitativa ou qualitativa ${ }^{15}$, a precisão desejada, o arbítrio do pesquisador e os custos compatíveis para realização do estudo ${ }^{14}$. Além disso, é muito importante que o pesquisador leve em conta todos os dados previamente encontrados, a verba e a infra-estrutura disponíveis, bem como a questão ética ${ }^{26}$. 
Tabela 4 - Média, desvio padrão e coeficiente de variação do índice CPOD em diferentes anos nas cidades de Bauru e Piracicaba.

Table 4 - Mean, standard deviation and coefficient of variation of the DMFT index in different periods of time in the cities of Bauru and Piracicaba.

\begin{tabular}{|c|c|c|c|c|c|c|}
\hline Erro amostral & Cidade & Ano & Média & $\mathrm{Dp}$ & CV\% & $\begin{array}{c}\text { Tamanho da } \\
\text { amostra }\end{array}$ \\
\hline \multirow[t]{7}{*}{$1 \%$} & Bauru & 1976 & 9,9 & 4,0 & 40,0 & 2.647 \\
\hline & & 1984 & 7,0 & 3,8 & 54,4 & 3.220 \\
\hline & & 1990 & 4,3 & 3,1 & 72,6 & 3.432 \\
\hline & & 1995 & 4,3 & 3,5 & 80,2 & 4.769 \\
\hline & & 2001 & 1,5 & 2,1 & 135,3 & 5.257 \\
\hline & Piracicaba & 2001 & 1,7 & 2,1 & 121,8 & 5.575 \\
\hline & & 2005 & 1,3 & 1,9 & 143,9 & 5.654 \\
\hline \multirow[t]{7}{*}{$2 \%$} & Bauru & 1976 & 9,9 & 4,0 & 40,0 & 1.609 \\
\hline & & 1984 & 7,0 & 3,8 & 54,4 & 2.261 \\
\hline & & 1990 & 4,3 & 3,1 & 72,6 & 2.736 \\
\hline & & 1995 & 4,3 & 3,5 & 80,2 & 3.698 \\
\hline & & 2001 & 1,5 & 2,1 & 135,3 & 4.727 \\
\hline & Piracicaba & 2001 & 1,7 & 2,1 & 121,8 & 4.861 \\
\hline & & 2005 & 1,3 & 1,9 & 143,9 & 5.109 \\
\hline \multirow[t]{7}{*}{$5 \%$} & Bauru & 1976 & 9,9 & 4,0 & 40,0 & 430 \\
\hline & & 1984 & 7,0 & 3,8 & 54,4 & 733 \\
\hline & & 1990 & 4,3 & 3,1 & 72,6 & 1.130 \\
\hline & & 1995 & 4,3 & 3,5 & 80,2 & 1.438 \\
\hline & & 2001 & 1,5 & 2,1 & 135,3 & 2.771 \\
\hline & Piracicaba & 2001 & 1,7 & 2,1 & 121,8 & 2.564 \\
\hline & & 2005 & 1,3 & 1,9 & 143,9 & 3.052 \\
\hline \multirow[t]{7}{*}{$10 \%$} & Bauru & 1976 & 9,9 & 4,0 & 40,0 & 119 \\
\hline & & 1984 & 7,0 & 3,8 & 54,4 & 215 \\
\hline & & 1990 & 4,3 & 3,1 & 72,6 & 376 \\
\hline & & 1995 & 4,3 & 3,5 & 80,2 & 452 \\
\hline & & 2001 & 1,5 & 2,1 & 135,3 & 1.118 \\
\hline & Piracicaba & 2001 & 1,7 & 2,1 & 121,8 & 954 \\
\hline & & 2005 & 1,3 & 1,9 & 143,9 & 1.252 \\
\hline
\end{tabular}

O presente estudo calculou o tamanho da amostra baseado na prevalência e distribuição da cárie dentária, que atualmente apresenta grande variabilidade entre as crianças. Pode ser constatado nos levantamentos realizados nos municípios de Bauru e Piracicaba, no decorrer dos anos, diminuição da média do CPO-D, aumento do desvio padrão e aumento do coeficiente de variação, significando dessa forma maior dispersão dos valores encontrados em relação à média. Isto implicou em considerável aumento das amostras necessárias para a realização de levantamentos quando considerado erro amostral de $1 \%$ e $2 \%$. Estes resultados confirmam o fato de que quanto menos se permite errar em relação ao verdadeiro valor da média da população, maior o tamanho da amostra e vice-versa. No presente estudo, um erro 
Tabela 5 - Cálculo do tamanho da amostra para o exame de cárie dental nas cidades de Bauru e Piracicaba e custos (em Reais) envolvidos com o levantamento.

Table 5 -Sample size calculation for dental caries surveys in the cities of Bauru and Piracicaba and the costs (R\$) related to these surveys.

\begin{tabular}{lcccccc}
\hline Cidade & Erro & Amostra & Custo & \multicolumn{2}{c}{ Custo variável } & \multirow{2}{*}{ Total } \\
\cline { 5 - 6 } & amostral & & fixo & Recursos Humanos & Material de consumo nn & \\
\hline Bauru & $1 \%$ & 5.257 & $1.456,58$ & $19.478,94$ & $6.525,69$ & $27.461,21$ \\
& $2 \%$ & 4.727 & $1.456,58$ & $17.515,11$ & 5.867 .78 & $24.839,47$ \\
& $5 \%$ & 2.771 & $1.456,58$ & $10.267,48$ & $3.439,74$ & $15.163,80$ \\
& $10 \%$ & 1.118 & $1.456,58$ & $4.142,56$ & $1.387,81$ & $6.986,95$ \\
\hline Piracicaba & $1 \%$ & 5.654 & $1.456,58$ & $20.949,95$ & $7.018,50$ & $29.425,03$ \\
& $2 \%$ & 5.109 & $1.456,58$ & $18.930,55$ & $6.341,97$ & $26.729,10$ \\
& $5 \%$ & 3.052 & $1.456,58$ & $11.308,68$ & $3.788,55$ & $16.553,81$ \\
\hline
\end{tabular}

de $10 \%$ torna-se aceitável, porque dentro de um perfil epidemiológico com índice CPO-D baixo, errar em média 0,2 dentes na média do CPO-D no município de Bauru, e 0,1 dentes em relação à média do CPO-D em Piracicaba não se afasta muito da verdadeira estimativa populacional. É importante ressaltar a limitação deste estudo frente às amostras selecionadas, já que as características de construção das mesmas, bem como os processos de execução, podem ser distintos, principalmente em relação aos estudos mais antigos.

O projeto SB-Brasil, em $2003^{27}$, seguiu, como técnica para estimar o tamanho da amostra, a frequência da doença na população e, para as idades que não se tinham dados, considerou-se proporção de 50\% com doença e margem de erro $10 \%$. No projeto SB Brasil 2010, os autores relatam que, sendo o CPO-D uma variável quantitativa, utilizou-se a média e o desvio padrão encontrados no levantamento de 2003 para o cálculo do tamanho da amostra para o levantamento de $2010^{10}$. Conforme foi verificado no Projeto SB-Brasil, e de acordo com os achados do presente estudo, há a necessidade do aumento do tamanho da amostra com a finalidade de se encontrar a doença na população, quando se observa a diminuição do valor da média do índice CPO-D. Levantamentos que foram realizados em anos diferentes para efeito de comparação da prevalência da cárie destacaram diminuição do índice da doença e amostras maiores com o passar dos anos. Em estudos conduzidos na França, as amostras foram de 1.000 crianças em 1987, 1.331 em 1993 e 6.000 em 19983; na Alemanha, foram de 18.459 em 1994-1995, 22.980 em 1997 e 24.679 em $2000^{4}$. O tamanho da amostra varia em relação à população total de um determinado município, no entanto, direcionando o estudo para prevalência, dentro desse novo perfil de redução da doença, há a necessidade de amostras cada vez maiores.

Se por um lado a metodologia para investigação epidemiológica é conhecida e especificada ${ }^{13}$, os estudos relacionados aos custos para sua realização são escassos. Estudos recentes na área médica têm mostrado preocupação em estimar e avaliar gastos em procedimentos na área da saúde dentro do setor público, levando em consideração materiais utilizados e mão-de-obra empregada ${ }^{21,22}$. O presente estudo considerou como material permanente os instrumentais recomendados pela OMS (sonda ball-point/IPC e espelho bucal), os materiais de consumo (luvas, máscara, álcool, gaze etc.) e a mão-de-obra composta por Cirurgião-Dentista e Auxiliar de Consultório Dentário (ACD). Pôde ser observado um aumento no tamanho das amostras ao longo dos anos e os custos relativos ao levantamento variaram razoavelmente quando considerados diferentes erros amostrais. Cabe lembrar que os levantamentos são 
realizados no mínimo a cada $5 \operatorname{anos}^{12}$, e os municípios recebem do Ministério da Saúde um valor per capita de acordo com seu número de habitantes, para empregar em atividades de promoção e prevenção em saúde ${ }^{28}$.

A importância de levantamentos epidemiológicos de cárie dentária se dá porque por meio dos mesmos é possível o acompanhamento da tendência da doença na população, além de permitir a verificação das estratégias aplicadas pelo sistema de saúde ${ }^{29}$.

O gestor que tenha como objetivo avaliar a prevalência de cárie na sua cidade deve levar em consideração, além do tamanho da amostra, o custo desse levantamento. $\mathrm{O}$ tamanho da amostra é calculado a partir do conhecimento da média e do desvio padrão estimados para aquela população em estudos anteriores ou em estudos pilotos, e o custo é calculado considerando-se a hora de trabalho dos recursos humanos necessários, somada aos custos dos materiais de consumo e permanente, como está demonstrado no presente artigo.

Baseado nos levantamentos epidemiológicos anteriormente realizados e que demonstraram haver a diminuição da média do índice CPO-D na população estudada, conclui-se que essa diminuição determina um aumento no tamanho das amostras, sendo que isso terá impacto nos custos e no tempo necessário para o desenvolvimento do estudo.

\section{Aspectos Éticos}

Não há conflito de interesse no presente estudo e o mesmo foi aprovado pelo Comitê de Ética em pesquisa da Faculdade de Odontologia de Piracicaba - UNICAMP sob o protocolo $n^{\circ}$. 104/2006.

\section{Referências}

1. Narvai PC, Castellanos R, Frazão P. Prevalência de cárie em dentes permanentes de escolares do Município de São Paulo, SP, 1970-1996. Rev Saude Publica 2000; 34: 196-200.

2. Aoba T, Fejerskov O. Dental fluorosis: chemistry and biology. Crit Rev Oral Biol Med 2002; 13: 155-70.

3. Bourgeois DM, France L, Roland E, France N, Desfontaine J. Caries prevalence 1987-1998 in 12-yearolds in France. Int Dental J 2004; 54: 193-200.

4. Pieper K, Schulte AG. The decline in dental caries among 12-year-old children in Germany between 1994 and 2000. Community Dent Health 2004; 21: 199-206.

5. Bastos JLD, Nomura LH, Peres MC. Tendência de cárie dentária em escolares de 12 e 13 anos de idade de uma mesma escola no período de 1971 a 2002, em Florianópolis, Santa Catarina, Brasil. Cad Saude Publica 2004; 20: 117-22.

6. Bastos RS, Olympio KPK, Bijella VT, Buzalaf MAR, Bastos JRM. Trends in dental caries prevalence in 12-year-old schoolchildren between 1976 and 2001 in Bauru, Brazil. Public Health 2005; 119:269-75.

7. Brasil. Ministério da Saúde. Levantamento epidemiológico em saúde bucal: Brasil, zona urbana, 1986. Brasília: Centro de Documentação, Ministério da Saúde; 1988.
8. Brasil. Ministério da Saúde. Levantamento epidemiológico em saúde bucal: Cárie Dental, Capitais; 1996. Área técnica de saúde bucal; 1996. Disponível em: http:// www.saude.gov.br [Acessado em 19 de janeiro de 2008]

9. Brasil. Ministério da Saúde. Projeto SB Brasil 2003: Condições de saúde bucal da população brasileira 2002-2003-resultados principais. Brasília: Coordenação de Saúde Bucal; 2004.

10. Brasil. Ministério da Saúde. Secretaria de Atenção à Saúde. Departamento de Atenção Básica. Coordenação Nacional de Saúde Bucal. SB Brasil 2010: Pesquisa Nacional de Saúde Bucal. Projeto Técnico. Brasília, 2009.

11. Tickle M. The 80:20 phenomenon: help or hindrance to planning caries prevention programmes? Community Dent Health 2002; 19: 39-42.

12. Pereira SM, Tagliaferro EPS, Ambrosano GMB, Cortellazzi KL, Meneghim MC, Pereira AC. Dental caries in 12-year-old schoolchildren and its relationship with socioeconomic and behavioral. Oral Health Prev Dent 2007; 5: 299-306.

13. Organização Mundial de Saúde. Levantamento epidemiológico básico de saúde bucal: manual de instruções. 3a ed. São Paulo: Santos; 1991. 
14. Luiz RR, Magnanini MMF. A lógica da determinação do tamanho da amostra em investigações epidemiológicas. Cad Saude Coletiva 2000; 8: 9-28.

15. Pandey RM. Approaches to sample size calculation in comparative studies. Indian J Pediatr 1999; 66: 533-8.

16. Carneiro AV. Cálculo da dimensão da amostra em estudos clínicos: princípios metodológicos básicos. Rev Port Cardiol 2003; 22: 1513-21.

17. Bastos JLD, Nomura LH, Peres MC. Dental pain, socioeconomic status, and dental caries in young male adults from southern Brazil. Cad Saude Publica 2005; 21 : 1416-23.

18. Ribeiro AG, Oliveira AF, Rosenblatt A. Cárie precoce na infância: prevalência e fatores de risco em pré-escolares, aos 48 meses, na cidade de João Pessoa, Paraíba, Brasil. Cad Saude Publica 2005; 21: 1695-700.

19. Roncalli AG, Unfer B, Costa ICC, Arcieri RM, Guimarães LOC, Saliba NA. Levantamentos epidemiológicos em saúde bucal: análise da metodologia proposta pela Organização Mundial de Saúde. Rev Bras Epidemiol 1998; 1: 177-89.

20. Roncalli AG. Aspectos Técnico-operacionais de um levantamento epidemiológico em saúde bucal. In: Ferreira MAF, Roncalli AG, Lima KC. Saúde bucal coletiva: conhecer para atuar. Natal: EDUFRN; 2005.

21. Costa JG, Santos AC, Rodrigues LC, Barreto ML, Roberts JA. Tuberculose em Salvador: custos para o sistema de saúde e para as famílias. Rev Saude Publica 2005; 39: 122-8.
22. Haddad N, Bittar E, Marchi AF, Kantorowitz CSV, Ayoub AC, Fonseca ML et al. Custos hospitalares da cirurgia de revascularização do miocárdio em pacientes coronarianos eletivos. Arq Bras Cardiol 2007; 88: 418-23.

23. World Health Organization. Oral health surveys: basic methods. 4.ed. Geneva: WHO; 1997.

24. Brasil. Ministério do Planejamento, Orçamento e Gestão. Instituto Brasileiro de Geografia e Estatística IBGE. Disponível em: http://www.ibge.gov.br [Acessado em 19 de janeiro de 2008]

25. Narvai PC, Frazão P, Roncalli AG, Antunes JLF. Cárie dentária no Brasil: declínio, polarização, iniquidade e exclusão social. Rev Panam Salud Publica 2006; 19: 38593.

26. Gogtay NJ. Principles of sample size calculation. Indian J Ophthalmol 2010 ; 58: 517-8.

27. Roncalli AG, Frazão P, Pattussi MP, Araújo IC, Ely HC, Batista SM. Projeto SB2000: uma perspectiva para a consolidação da Epidemiologia em Saúde Bucal Coletiva. Rev Bras Odontol Saude Coletiva 2000; 1: 9-25.

28. Aerts D, Abegg C, Cesa K. O papel do cirurgião-dentista no Sistema Único de Saúde. Cienc Saude Coletiva 2004; 9: 131-8.

29. Reis SCGB, Freire MCM, Higino MASP, Batista SMO, Rezende KLV, Queiroz MG. Declínio de cárie em escolares de 12 anos da rede pública de Goiânia, Goiás, Brasil, no período de 1988 a 2003. Rev Bras Epidemiol 2009; 12: 92-8.

Recebido em: 03/06/11

Versão final apresentada em: 19/01/12

Aprovado em: 03/02/12 